\title{
The effect of the type of the bariatric surgery in the lipid profile: an age, sex, body mass index, and excess weight loss matched study
}

SÃO JOÃO

H O S P I T A L

Cunha $\mathrm{FM}^{1,2}$, Oliveira $\mathrm{J}^{1,2}$, Preto $\mathrm{J}^{3}$; Saavedra $\mathrm{A}^{1}$, Costa $\mathrm{MM}^{1}$, Lau $\mathrm{E}^{1,2}$, Magalhães $\mathrm{D}^{1}$, Bettencourt-Silva $\mathrm{R}^{1}$, Freitas $\mathrm{P}^{1,2}$, Varela $\mathrm{A}^{1,2}$, Carvalho $\mathrm{D}^{1,2}$

${ }^{1}$ Serviço de Endocrinologia, Diabetes e Metabolismo do Centro Hospitalar de São João EPE; ${ }^{2}$ Faculdade de Medicina da Universidade do Porto; ${ }^{3}$ Serviço de Cirurgia Geral do Centro Hospitalar de São João

\section{Background}

Dyslipidemia is common in obese patients. High levels of triglycerides (TG), and low density lipoprotein (LDL) cholesterol, and low levels of high density lipoprotein (HDL) cholesterol are frequently encountered in obesity.

Bariatric surgery improves the lipid profile. A still unanswered question is whether this improvement is merely weight dependent or also results from factors inherent to specificities of the bariatric procedure itself.

We aimed to study the lipid profile 1 year after bariatric surgery and compare its changes between the different procedures in patients matched for age, sex, initial weight and for excess weight loss (EWL)

\section{Methods}

Retrospective study of morbidly obese patients submitted to bariatric surgery (AGB: adjustable gastric banding, RYGB: Roux-en-Y gastric bypass and SG: sleeve gastrectomy) between 2010 and 2012

Body mass index (BMI): weight $(\mathrm{Kg}) /$ height $^{2}(\mathrm{~m})$

EWL: [(excess weight - weight lost at 1 year)/excess weight] x 100

Type 2 Diabetes mellitus was defined by a fasting glucose $\geq 126 \mathrm{mg} / \mathrm{dL}$, a 2-hour plasma glucose $\geq 200 \mathrm{mg} / \mathrm{dL}$ during a $75 \mathrm{~g}$ oral glucose tolerance test, a $\mathrm{HbAl} \geq 6.5 \%$, or the use of oral hypoglycaemic agents or insulin.

Glomerular filtration rate was estimated using the Modification of Diet in Renal Disease formula

TC, HDL and TG were measured directly in the plasma, LDL was calculated using the Friedwald formula.

Percentage of variation $(\% \Delta)$ of each of the lipid profile parameters: [(presurgical value - value at 1 year)/ pre-surgical value] x 100; except for HDL $=[$ (value at 1 year - pre-surgical value $) /$ pre-surgical value $] \times 100$.

Patients were matched for age ( 5years), sex, pre-surgery BMI ( 2 $\left.\mathrm{Kg} / \mathrm{m}^{2}\right)$ and EWL ( $\left.5 \%\right)$.

The baseline characteristics were compared: $\chi^{2}$ for categorical variables, One-way ANOVA for normally distributed continuous variables, and Kruskal-Wallis test for skewed continuous variables.

Comparison between baseline and 1-year lipid profile, its variation $(\Delta)$ and $\% \Delta$ : paired samples $t$ test for normally distributed continuous variables and Wilcoxon test for non-normally distributed continuous variables.

\section{Results}

Median age: 41 (35-52) years; $11.8 \%$ were male. BMI at the time of surgery: $44.0 \pm 4.6 \mathrm{Kg} / \mathrm{m}^{2}$ and $32.1 \pm 4.4 \mathrm{Kg} / \mathrm{m}^{2}$ at 1 year. EWL at 1 year: $64.2 \pm 18.9 \%$.

Table 1 - Baseline characteristics of patients submitted to bariatric surgery

\begin{tabular}{lccccc}
\hline & $\begin{array}{c}\text { All } \\
(\mathrm{n}=229)\end{array}$ & $\begin{array}{c}\text { AGB } \\
(\mathrm{n}=80)\end{array}$ & $\begin{array}{c}\text { RYGB } \\
(\mathrm{n}=94)\end{array}$ & $\begin{array}{c}\text { SG } \\
(\mathrm{n}=55)\end{array}$ & $\mathrm{p}$ \\
\hline Statins, n (\%) & $50(22.0)$ & $14(17.7)$ & $23(24.7)$ & $13(23.6)$ & 0.51 \\
Statins 1 year, n (\%) & $31(15.0)$ & $10(14.3)$ & $8(9.1)$ & $13(23.6)$ & $\mathbf{0 . 0 2}$ \\
Fibrate, n (\%) & $14(6.2)$ & $5(6.3)$ & $7(7.5)$ & $2(3.6)$ & 0.64 \\
Fibrate 1 year, n (\%) & $2(1.0)$ & $1(1.4)$ & $1(1.1)$ & $0(0.0)$ & 0.72 \\
T2DM, n (\%) & $48(21.0)$ & $17(21.3)$ & $22(23.4)$ & $9(16.4)$ & 0.59 \\
Pre-diabetes, n (\%) & $107(46.7)$ & $36(45.0)$ & $44(46.8)$ & $27(49.1)$ & 0.90 \\
HbA1c, \% & $5.7(5.4-6.0)$ & $5.7(5.4-6.0)$ & $5.7(5.4-6.1)$ & $5.6(5.3-6.0)$ & 0.46 \\
HbA1c 1 year, \% & $5.3(5.1-5.6)$ & $5.3(5.1-5.5)$ & $5.3(5.1-5.7)$ & $5.3(5.0-5.6)$ & 0.66 \\
Hypertension, n (\%) & $94(41.4)$ & $30(38.0)$ & $44(47.3)$ & $20(36.4)$ & 0.32
\end{tabular}

$\mathrm{TSH}(\mathrm{mIU} / \mathrm{L}) \quad 1.77(1.34-2.59) \quad 1.68(1.22-2.54) 1.99(1.36-2.82) \quad 1.63(1.36-2.54) 0.22$ GFR ( $\mathrm{mL} / \mathrm{min})$ $86 \pm 21$ $82 \pm 17$ $85 \pm 21$ $90 \pm 25 \quad 0.02$

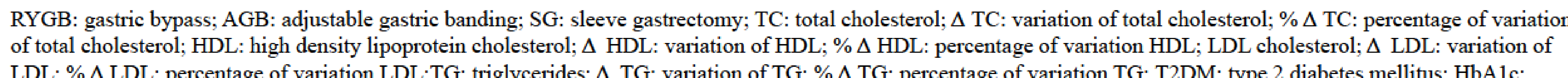
LDL; \% $\Delta$ LDL: percentage of variation LDL; TG: triglycerides; $\Delta$ TG: variation of TG; \% $\Delta$ TG: percentage of variation TG; T2DM: type 2 diabetes
glycated haemoglobin; TSH: thyroid-stimulating hormone; GFR: glomerular filtration rate. Mean standard deviation; median (interyuntile ranes).
Table 2 - Comparison of the lipid profile between the various bariatric surgery types in age-, sex-, BMI-, EWL-matched patients

\begin{tabular}{lccc}
\hline & RYGB $(\mathbf{n}=\mathbf{7 2})$ & AGB $(\mathbf{n}=\mathbf{7 2})$ & $\mathbf{p}$ \\
\hline TC, $\mathrm{mg} / \mathrm{dL}$ & $208 \pm 40$ & $200 \pm 39$ & 0.21 \\
TC 1 year, mg/dL & $185 \pm 31^{*}$ & $193 \pm 44$ & 0.17 \\
$\Delta$ TC, mg/dL & $23 \pm 37$ & $7 \pm 44$ & $\mathbf{0 . 0 0 8}$ \\
\% $\mathrm{TC}$ & $9.1 \pm 17.9$ & $1.8 \pm 20.0$ & $\mathbf{0 . 0 1}$ \\
\hline HDL, mg/dL & $50 \pm 11$ & $51 \pm 11$ & 0.52 \\
HDL 1 year, mg/dL & $59 \pm 13^{*}$ & $56 \pm 10^{*}$ & 0.15 \\
$\Delta$ HDL, mg/dL & $9 \pm 10$ & $5 \pm 10$ & $\mathbf{0 . 0 3}$ \\
$\% \Delta$ HDL & $19.3 \pm 21.7$ & $13.0 \pm 22.8$ & 0.10 \\
\hline LDL, mg/dL & $135 \pm 32$ & $124 \pm 32$ & 0.06 \\
LDL 1 year, mg/dL & $108 \pm 25^{*}$ & $118 \pm 37$ & $\mathbf{0 . 0 5}$ \\
$\Delta$ LDL, mg/dL & $27 \pm 32$ & $10 \pm 35$ & $<\mathbf{0 . 0 0 1}$ \\
$\% \Delta$ LDL, mg/dL & $17.2 \pm 25.0$ & $3.2 \pm 26.0$ & $<\mathbf{0 . 0 0 1}$ \\
\hline TG, mg/dL & $144 \pm 72$ & $149 \pm 77$ & 0.54 \\
TG 1 year, mg/dL & $95 \pm 36^{*}$ & $98 \pm 48^{*}$ & 0.91 \\
$\Delta$ TG, mg/dL & $49 \pm 61$ & $49 \pm 61$ & 0.79 \\
$\% \Delta$ TG & $26.7 \pm 28.4$ & $26.7 \pm 28.4$ & 0.15 \\
\hline
\end{tabular}

\begin{tabular}{lccc}
\hline & RYGB $(\mathbf{n}=\mathbf{4 7})$ & SG $(\mathbf{n}=\mathbf{4 7})$ & $\mathbf{p}$ \\
\hline TC, mg/dL & $200 \pm 39$ & $204 \pm 43$ & 0.64 \\
TC 1 year, mg/dL & $182 \pm 31^{*}$ & $200 \pm 34$ & $\mathbf{0 . 0 2}$ \\
$\Delta$ TC, mg/dL & $18 \pm 30$ & $4 \pm 36$ & 0.06 \\
$\% \Delta$ TC & $7.3 \pm 14.8$ & $-0.3 \pm 18.8$ & $\mathbf{0 . 0 3}$ \\
\hline HDL, mg/dL & $50 \pm 11$ & $50 \pm 11$ & 0.87 \\
HDL 1 year, mg/dL & $60 \pm 12^{*}$ & $58 \pm 12^{*}$ & 0.44 \\
$\Delta$ HDL, mg/dL & $10 \pm 9$ & $8 \pm 11$ & 0.31 \\
\% $\Delta$ HDL & $22.1 \pm 20.5$ & $18.3 \pm 26.9$ & 0.47 \\
LDL, mg/dL & $126 \pm 33$ & $128 \pm 33$ & 0.71 \\
LDL 1 year, mg/dL & $105 \pm 27 *$ & $124 \pm 31$ & $\mathbf{0 . 0 0 4}$ \\
$\Delta$ LDL, mg/dL & $21 \pm 28$ & $4 \pm 33$ & $\mathbf{0 . 0 2}$ \\
\% $\Delta$ LDL, mg/dL & $14.1 \pm 21.1$ & $-1.2 \pm 28.2$ & $\mathbf{0 . 0 0 7}$ \\
\hline TG, mg/dL & $139 \pm 54$ & $130 \pm 55$ & 0.44 \\
TG 1 year, mg/dL & $88 \pm 34 *$ & $92 \pm 37 *$ & 0.67 \\
$\Delta$ TG, mg/dL & $50 \pm 55$ & $38 \pm 46$ & 0.26 \\
$\% \Delta$ TG & $30.3 \pm 29.4$ & $23.9 \pm 28.0$ & 0.29 \\
\hline
\end{tabular}

\begin{tabular}{|c|c|c|c|}
\hline & AGB $(n=33)$ & SG $(n=33)$ & $\mathbf{p}$ \\
\hline $\mathrm{TC}, \mathrm{mg} / \mathrm{dL}$ & $198 \pm 46$ & $211 \pm 46$ & 0.20 \\
\hline TC 1 year, mg/dL & $181 \pm 61$ & $216 \pm 46$ & 0.02 \\
\hline$\Delta \mathrm{TC}, \mathrm{mg} / \mathrm{dL}$ & $5 \pm 45$ & $-4 \pm 38$ & 0.33 \\
\hline$\% \Delta \mathrm{TC}$ & $3.0 \pm 24.3$ & $-4.2 \pm 20.4$ & 0.19 \\
\hline $\mathrm{HDL}, \mathrm{mg} / \mathrm{dL}$ & $51 \pm 10$ & $49 \pm 10$ & 0.59 \\
\hline HDL 1 year, mg/dL & $53 \pm 16$ & $57 \pm 12 *$ & 0.34 \\
\hline$\Delta \mathrm{HDL}, \mathrm{mg} / \mathrm{dL}$ & $6 \pm 10$ & $8 \pm 7$ & 0.53 \\
\hline$\% \Delta \mathrm{HDL}$ & $20.8 \pm 35.0$ & $16.3 \pm 16.5$ & 0.52 \\
\hline $\mathrm{LDL}, \mathrm{mg} / \mathrm{dL}$ & $123 \pm 39$ & $133 \pm 35$ & 0.22 \\
\hline LDL 1 year, mg/dL & $111 \pm 44$ & $140 \pm 41$ & 0.02 \\
\hline$\Delta \mathrm{LDL}, \mathrm{mg} / \mathrm{dL}$ & $5 \pm 36$ & $-7 \pm 35$ & 0.21 \\
\hline$\% \Delta \mathrm{LDL}, \mathrm{mg} / \mathrm{dL}$ & $5.6 \pm 39.1$ & $-8.3 \pm 29.7$ & 0.12 \\
\hline $\mathrm{TG}, \mathrm{mg} / \mathrm{dL}$ & $149 \pm 72$ & $141 \pm 78$ & 0.65 \\
\hline TG 1 year, mg/dL & $88 \pm 42^{*}$ & $97 \pm 42^{*}$ & 0.33 \\
\hline$\Delta \mathrm{TG}, \mathrm{mg} / \mathrm{dL}$ & $57 \pm 62$ & $44 \pm 54$ & 0.38 \\
\hline$\% \Delta \mathrm{TG}$ & $31.5 \pm 22.4$ & $24.7 \pm 29.4$ & 0.28 \\
\hline
\end{tabular}

\section{Conclusions}

RYGB is the only bariatric surgery that reduces TC and LDL cholesterol levels in age-, sex-, BMI-, and EWL-matched patients.

All 3 procedures improved TG and HDL similarly when the confounding effect of weight loss is eliminated.

Improvements in HDL and TG are probably attributable to weight loss. Decreases in LDL and TC appear not to be completely attributed to weight reduction; factors inherent to the specific surgical procedure may play a role. 